\title{
A prospective study to assess the safety and expulsion rate of copper T380A in immediate post-partum period during caesarean section
}

\author{
Preet Kamal Bedi, Manjit Singh Guliani*, Suman Bala
}

Department of Obstetrics and Gynaecology, GMC, Amritsar, Punjab, India

Received: 27 June 2016

Revised: 11 August 2016

Accepted: 12 August 2016

\section{*Correspondence:}

Dr. Manjit Singh Guliani,

E-mail: m.s.guliani60@gmail.com

Copyright: () the author(s), publisher and licensee Medip Academy. This is an open-access article distributed under the terms of the Creative Commons Attribution Non-Commercial License, which permits unrestricted non-commercial use, distribution, and reproduction in any medium, provided the original work is properly cited.

\begin{abstract}
Background: Immediate post-partum IUCD insertion deserves great attention as it can provide immediate contraception and prevents repeated unintended pregnancies. Increasing number of women undergoing elective cesarean section due to repeat cesarean section need effective long term contraception to allow them recuperate from surgery and for reliable means of child spacing. The postpartum insertion of an IUCD provides a convenient opportunity for the woman to receive IUCD services. This is particularly important for women who have limited access to medical care. The objective of this study was to assess the safety in terms of perforation, pain, bleeding, foul smelling vaginal discharge and expulsion rates at 6 weeks follow up and reasons for removal/discontinuation willingness to continue when CuT380A is inserted immediately after C-section deliveries.

Methods: The present study was conducted at Bebe Nanaki Mother and Child Care Hospital, Govt. Medical College, Amritsar, between period February 2014 to June 2015, on 200 cases undergoing caesarean section delivery willing for IUCD insertion and who fulfilled the medical eligibility criteria and had no contraindications for PPIUCD and patients were followed up at 6 weeks.

Results: A total of 200 women underwent postpartum intracaesarean insertion of copper T 380A. And 16 women lost to follow up. Acceptance was more in the multigravida. The mean age of women included in the study was $25.65 \pm 2.42$ years. Most common post-insertion complication observed in the immediate postoperative period was excessive bleeding. The common adverse events observed during follow-up of 6 weeks were menstrual complaints, excessive vaginal discharge and persistent pelvic pain. At the end 6 weeks, there were 6 expulsions, 4 removals, and gross cumulative expulsion, removal, failure and continuation rates of 3.2\%, 2.2\% and 5.4\%, respectively.

Conclusions: Inserting CuT380A after placental delivery during cesarean section is safe and effective, has high retention rate. The expulsion rate was not high, and can be further reduced with practice. The method may be particularly beneficial in our setting where women do not come for post natal contraception counseling and usage. The government needs to develop strategies to promote PPIUCD by increasing awareness, by arranging trainings among healthcare providers.
\end{abstract}

Keywords: IUCD, PPIUCD, CuT380A

\section{INTRODUCTION}

Immediate post-partum IUCD insertion deserves great attention as it can provide immediate contraception and prevents repeated unintended pregnancies.
Increasing number of women undergo elective caesarean section due to repeat caesarean section, breech presentation, fetal or maternal conditions, these women need effective long term contraception to allow them recuperate from surgery and for reliable means of child spacing. Studies show that pregnancies taking place within 24 months of a previous birth have a higher risk of 
adverse outcomes like abortions, premature labor, low birth weight babies, fetal loss, postpartum haemorrhage and maternal death. In India, 65 per cent of women in the first year post-partum have an unmet need for family planning. Hence contraception needs to be practiced in this critical period. ${ }^{1}$

Insertion of an intrauterine device (IUD) immediately after delivery is appealing for several reasons. The woman is known not to be pregnant, her motivation for contraception may be high, and the setting may be convenient for both the woman and her provider. The postpartum insertion of an IUCD provides a convenient opportunity for the woman to receive IUCD services, who have limited access to medical care

Intrauterine contraceptive devices (IUCD) to prevent pregnancy are among the oldest methods of contraception. The modern IUCD is a highly effective, safe, private, long-acting, coitus independent and rapidly reversible method of contraception with few side effects. Intrauterine contraception is the most cost-effective method of contraception today. Many women also find the IUCD to be very convenient because it requires little action once it is in place.

In India, Copper T 380A is being supplied free of cost by the government, to all health centres and private practitioners. This device is a proven highly effective and reversible spacing method of interval contraception, with effective protection for 10 years. However, the device has not attained much popularity due to the myths and misconceptions amongst the general public and health care personnel. Besides, due to the fear of perforation and infection, and also, lack of proper training, most health care providers are reluctant in performing interval IUCD insertion in women with previous caesarean delivery.

This technique offers the obstetrician an opportunity to insert the IUCD into the uterus under vision, thus obviating the fear of perforating the uterus during the procedure. Not only is IUCD high effective but also has immediate effectiveness, long term protection and immediate return to fertility upon removal. Other benefits include; non-interference with intercourse can be used by lactating women and helps prevent ectopic pregnancy.

\section{METHODS}

The present study was conducted at Bebe Nanaki Mother and Child Care Hospital, Govt. Medical College, Amritsar between period February 2014 to June 2015, on 200 cases undergoing caesarean section delivery, willing for IUCD insertion and fulfilling WHO standard medical criteria for PPIUCD insertion. Counseling for PPIUCD (post-partum intrauterine contraceptive device) was done as a part of study either during their antenatal visits or early labour hours or prior to a scheduled caesarean section
After the placenta and membranes were removed, IUCD was introduced through the uterine incision during a caesarean section and placed at the uterine fundus. This was done manually or using a regular ring forceps, since it is not necessary to use a long instrument to reach the fundus at time of LSCS. No attempt was done to pass the strings of the IUCD through the cervical os before closure of the uterus as this can displace the IUCD and leave it lower down in the uterine cavity. Women in whom the PPIUCD was inserted were assessed before discharge and followed at six weeks after IUCD insertion. Upon discharge a PPIUCD follow up card was given which contained information of the date of insertion and follow up visit, type of IUCD inserted, date of expiry of the IUCD and the telephone number of the principal investigator. These women were also advised to come back any time if she had any concern or experiences any warning signs and symptoms or if the IUCD is expelled. At each visit, a detailed history regarding excessive bleeding, symptoms of infection, abdominal cramps or any other complaint was taken, along with general physical and pelvic examination. Ultrasonography was done if the IUCD thread was not visible.

\section{RESULTS}

Table 1: Sociodemographic and obstetric characteristics of the parturients included in study $(\mathrm{N}=\mathbf{2 0 0})$.

\begin{tabular}{|lll|}
\hline Characteristics & Number & Percentage (\%) \\
\hline Age groups (years) & \multicolumn{1}{l|}{} \\
\hline $20-25$ & 118 & 59 \\
\hline $25-30$ & 70 & 35 \\
\hline$>30$ & 12 & 6 \\
\hline Literacy & & \\
\hline Illiterate & 50 & 25 \\
\hline Primary & 54 & 27 \\
\hline Secondary & 80 & 40 \\
\hline Higher education & 16 & 8 \\
\hline Gravida & & \\
\hline Primigavida & 48 & 24 \\
\hline Multigravida & 152 & 76 \\
\hline Parity & & \\
\hline 0 & 50 & 25 \\
\hline 1 or $>1$ & 150 & 75 \\
\hline Last child birth & & \\
\hline $0-2$ & 108 & 54 \\
\hline 2-3 & 42 & 21 \\
\hline 3-4 & 42 & 21 \\
\hline$>5$ & 8 & 4 \\
\hline Future pregnancy desire & \\
\hline 1-2 years & 0 & 0 \\
\hline 3-5years & 44 & 22 \\
\hline$>5 y e a r s ~$ & 60 & 30 \\
\hline Not sure & 22 & \\
\hline Never & 54 & \\
\hline & & \\
\hline
\end{tabular}


Table 2: Reasons for acceptance of PPIUCD among parturients whom IUCD was inserted $(N=200)$.

\begin{tabular}{|lll|}
\hline Reason & No. & Percentage \\
\hline Long term & 118 & 59 \\
\hline Safe & 40 & 20 \\
\hline Reversible & 42 & 21 \\
\hline Fewer clinic visits & 56 & 28 \\
\hline
\end{tabular}

Table 3: Post insertion complications $(\mathrm{N}=\mathbf{2 0 0})$.

\begin{tabular}{|lll|}
\hline Complications & No. & Percentage \\
\hline Fever & 4 & 2 \\
\hline Excessive bleeding & 2 & 1 \\
\hline Pain & 2 & 1 \\
\hline
\end{tabular}

Table 4: Complications at 6 weeks after PPIUCD insertion $(\mathrm{N}=184)$.

\begin{tabular}{|lll|}
\hline Complications & No. & Percentage \\
\hline Pain & 14 & 7.6 \\
\hline Bleeding & 32 & 17.4 \\
\hline Pyrexia & 2 & 1.1 \\
\hline Discharge & 8 & 4.3 \\
\hline Perforation & 0 & 0 \\
\hline Expulsion & 6 & 3.2 \\
\hline Removed & 4 & 2.2 \\
\hline
\end{tabular}

Table 5: Continuation rate in the study.

\begin{tabular}{|lll|}
\hline Continuation rate & Number & Percentage \\
\hline Total insertion & 200 & \\
\hline Total follow up & 184 & \\
\hline Expulsion & 4 & 2.1 \\
\hline Removal & 6 & 3.2 \\
\hline Continuation & 174 & 94.5 \\
\hline
\end{tabular}

A total of 200 women fulfilling WHO standard medical criteria for PPIUCD insertion and willing to comply with the study protocol had post placental intracaesarean insertion of Copper T 380A. Out of 200 women 16 were lost to follow up.

The demographic and clinical profile of the women is shown in (Table 1). Acceptance of intrauterine contraceptive device was the most common among multigravida women $(76 \%)$ in case of primigavida it was (24\%). Majority of the women (54\%) had their last child birth less than 2 years ago. More than half $(59.0 \%)$ of those women who accepted PPIUCD were due to the reason of its long term effects (Table 2).

Only 4\% had post insertion complications (Table 3) Out of 184 parturients who were followed up after PPIUCD insertion, 52 (28.2\%) developed complications (Table 4). Out of 184 mothers who were still in the study at 6 weeks, there were 6 expulsions, 4 removals, and with gross cumulative expulsion, removal and discontinuation rates of $3.2 \%, 2.2 \%$ and $5.4 \%$ respectively.

\section{DISCUSSION}

This study was carried out to determine uptake and outcome of intra-operative IUCD placement together with assessing the success that is the continuation rate at the end of the puerperium in a cohort of mothers who underwent elective cesarean section and required a long term reversible method of contraception.

Majority of the women (75\%) in the study population had at least a primary level of education. Acceptance of the use of PPIUCD was higher among women with secondary education $(40 \%)$, than those with no formal education $(25 \%)$. This could be reasoned out that educated women are high achievers and have greater labor market opportunities than the less educated women. This was similar to a study done in Egypt by Safwat et al where women with no formal education had an acceptance of $9.4 \%$ while those with formal education acceptance was $19.4 \% .^{2}$ Education has a positive effect on modern contraceptive use as shown in a study done in Zimbabwe.

Acceptance of intrauterine contraceptive device was the most common among multigravida women $(76 \%)$. In case of primigravida it was $(24 \%)$, this finding is similar to that of the study by Grimes et al where they found higher acceptance in multiparous clients $(65.1 \%) .{ }^{3}$ The duration since last child birth was significantly associated with acceptance of PPIUCD. About 54\% of the PPIUCD acceptors had their last child birth less than 2 years ago. Women who came for first delivery with short pregnancy interval felt the need for a long acting and reliable methods of contraception. In a report released by WHO in 2006, better family planning and birth spacing services resulted in better maternal and neonatal outcome. When promoted in countries with high birth rates, $32 \%$ of all maternal deaths and over one million deaths of children fewer than 5 years could be prevented. Healthy timing and spacing of pregnancies have a positive effect on maternal health and new born outcomes. ${ }^{4}$ This finding in the study indicates towards a positive maternal health in future.

PPIUCD has distinct advantage. It is free from systemic side effects and does not affect breast feeding as seen with hormonal methods. It is a reversible method. PPIUCD does not require regular user compliance. It is also not coital dependent and there is no pain on insertion when used post-placentally at the time of LSCS.

Majority (72\%) of the study population were not aware of the PPIUCD. Among women who had the PPIUCD inserted, 58\% have ever heard about PPIUCD from the antenatal clinic. This could be because the PPIUCD is a relatively new method of contraception in this community. In a WHO report released in 2010, on unmet 
need for family planning, one of the common reasons for non-use of contraception included lack of awareness. Another possible explanation for this would be that only the health care workers in the antenatal clinics and postnatal wards were aware of PPIUCD.

Among women whom PPIUCD was inserted, more than half $(59 \%)$ accepted due to its long term effect, $20 \%$ due to its safety, $28 \%$ due to fewer clinic visits and $21 \%$ due to its reversibility. This shows that postpartum women need a contraceptive method which is long acting, safe, reversible and convenient.

Infectious morbidity in the present study was consistent with previous reports by Celen et al and Eroglu et al but lower than that reported by Bhutta et al. ${ }^{5-7}$ Fever was observed to be the most common post-insertion complication in 4 out of 200 women (2\%). It was due to superficial wound infection in 2 , and urinary tract infection in 2 subjects. Women with superficial wound infection responded to local treatment, urinary tract infection was observed in 2 women, of which only one was symptomatic with fever; woman responded to antibiotics. Only 2 cases of excessive bleeding were observed in the study responded to tranexamic acid.

Majority (52\%) of the women who were inserted with the PPIUCD had their future pregnancy desire of 3 to 5 years. In half of them the period was more than five years. This was due to its long acting and reversible effect. $37 \%$ of these women wanted to limit their pregnancies but accepted the PPIUCD. The PPIUCD is especially good for women who think they do not want any more children, but want to delay sterilization until they are certain. ${ }^{8}$

The common adverse events observed during follow up were menstrual complaints, excessive vaginal discharge and persistent pelvic pain. At the first follow up visit, post insertion bleeding or spotting was reported by $16.67 \%$ while $4.3 \%$ women had vaginal discharge. Culture of vaginal discharge was nonspecific. Pelvic pain in most of the women was relieved by analgesics. According to an ICMR study on urban women, pelvic pain is a common symptom reported in $25 \%$ users following interval IUCD insertion. ${ }^{9}$ All women diagnosed with pelvic infection in the present study, were treated successfully with antibiotics.

Visibility of strings is important as it assures both, the IUCD user and the health care worker about proper placement of the device, and provides ease of removal. In intracaesarean insertion, though at the time of insertion threads are not outside cervical os, involution of uterus makes them visible in most cases at the first visit; however in a few cases threads may get curled up and not be seen at external os. This may cause apprehension to the health care worker as missing strings may indicate expulsion, mal-positioning or perforation. 36 (19.56\%) among those inserted with PPIUCD had lost strings during follow up at 4-6 weeks. In 28 cases, strings were found in the cervical canal. Rest four cases needed ultrasound and confirmed that the IUCD was in situ. It should be noted that there were no serious complications in the study.

Like other studies bleeding $(19.56 \%)$ out numbers other complications. ${ }^{10}$ It is really worrying. But only 6 out of $36(16.67 \%)$ insisted on removal, rest retained IUCD with reassurance only, which speaks of the importance of positive attitude. Expulsion rates of the immediate PPIUCD at 4-6 weeks interval were $4(2.1 \%)$. This was similar to a multi-country study done in Belgium, Chile and Phillippines which showed the rate of expulsion at 1 month ranging from 4.6 to $16 \%$. $^{11}$ Removal rates are similar in clients having or not having complication $(3.2 \%)$. It speaks of the importance and motivation prior to insertion in continuing PPIUCD.

There were no cases of perforation or misplaced IUD in the present study. Global health technical briefs on immediate post-partum insertion safety and efficacy said that there are a few reports addressing the relative safety of immediate post-partum insertion. ${ }^{12}$

In the present study, there were no cases of PID. A study conducted in 13 countries studied infection (PID) due to IUD. They have reported similar rate of infection with immediate insertion and interval insertion. ${ }^{13}$ Another trial did not find any instance of infection due to post-partum IUD insertion. ${ }^{14}$

The success rate of intra-operative IUCD placement can be measured by the continuation rates which were at $94.5 \%$. These results are similar to studies which reported continuation rate of postpartum IUCD at $90.5 \%$ in 6 weeks and $80 \%$ in 6 months respectively. ${ }^{15,16} \mathrm{~A}$ regional study done in Uganda at Mulago hospital by Lester et al compared intra-cesarean insertion of the copper T 380A verses 6 week post cesarean insertion found that uptake was better in immediate intra-cesarean insertion compared to interval insertion with better continuation rate of $83 \%$ in immediate verses $53 \%$ in delayed insertion at 6 months. ${ }^{17}$

Longer duration of follow up to assess the rate of expulsion and discontinuation would have been informative in these patients. Follow up on the antenatal mothers who declined insertion would have also helped in assessing the relative risks of the mentioned outcomes.

\section{CONCLUSION}

The acceptability of intra-operative placement of IUCD in mothers undergoing elective caesarean section was high. Its uptake has been encouraging and its safety demonstrated. With highest retention rates compared to other timings and routes of insertion and low rate of discontinuation at 6 weeks. The method may be particularly beneficial in our setting where women do not 
come for post natal contraception counseling and usage. Majority of the women never heard about PPIUCD before admission to labour room.

We can conclude that inserting CuT 380A after placental delivery during caesarean section is safe and effective, has high retention rate. The expulsion rate was not high, and can be reduced further with practice.

With the high level of acceptance, despite low levels of awareness, the government needs to develop strategies to increase public awareness of the PPIUCD through different media sources. It is also important to give training on PPIUCD in order to increase knowledge and skills among health care providers. This will also further promote PPIUCD use and aid in reduction of the expulsion rates.

\section{Funding: No funding sources}

Conflict of interest: None declared

Ethical approval: The study was approved by the Institutional Ethics Committee

\section{REFERENCES}

1. Post-partum IUCD reference manual. New Delhi: Family Planning Division, Ministry of Health and Family Welfare, Government of India; 2010.

2. Safwat A, Mohamed Momen A, Kamel Omar M, Shaaban HT, Salem. Acceptability for the use of postpartum intrauterine contraceptive devices: Assuit Experience. Medical Principles and Practice. 2003;12:170-5.

3. Grimes D, Schulz K, van Vliet H, Stanwood N. Immediate post-partum insertion of intrauterine devices: a Cochrane review. Hum Reprod. 2001;17(3):549-54.

4. Maternal and Child Health Integrated Program and PPFP activities, WHO Report. Available at http://k4health.org/sites/default/files/PPFPMeetingR eport_formatted.pdf.

5. Çelen Sucak A, Yıldız Y, Danıman N. Immediate post-placental insertion of an intrauterine contraceptive device during cesarean section Contraception. 2011;84(3):240-3.

6. Eroglu K, Akkuzu G, Vural G, Dilbaz B, Akin A, Takin L, et al. Comparison of efficacy and complications of IUD insertion in immediate postplacental/ early postpartum period with interval period: 1 year follow-up. Contraception. 2006;74(5):376-81.

7. Bhutta SZ, Butt IJ, Bano K. Insertion of intrauterine contraceptive device at caesarean section $\mathrm{J}$ Coll Physicians Surg Pak. 2011;21(9):527-30.

8. Postpartum IUCD Reference Manual, New Delhi: Family Planning Division, Ministry of Health and Family Welfare, Government of India; 2010.

9. Indian council of Medical Research. Task force study on psycho-social factors affecting continuation and discontinuation of intrauterine device and oral pill in urban India. New Delhi: Indian Council of medical Research; 1986.

10. Celen S, Moroy P, Sucak A, Aktulay A, Danişman $\mathrm{N}$, et al. Clinical outcomes of early post placental insertion of intrauterine contraceptive devices. Contraception. 2004;69:279-82.

11. Blanchard H, Mac Kiag C. ACCESS-FP program. Postpartum contraception, 2006. Available at http://www.k4health.org/sites/default/files/postpartu mabortion_English.pdf.

12. Sofat R. Postpartum CuT insertion - a trial. Indian J Maternal Child Health. 1990;1:23-4.

13. Lopez, Grimes, Szpir (Family health International/CRTU 7. programme). Immediate postpartum insertion of an IUD is safe and effective. United States Agency for International Development and Family Health International); 2007. Stefan Welkovic, L Costa, A Faundes, R Ximenes, C Costa. Global health technical briefs. USA: USAID; 2007.

14. Welkovic S, Costa LO, Faúndes A, de Alencar Ximenes R, Costa CF. Postpartum bleeding and infection after postplacental IUD insertion. Contraception. 2001;63:155-8.

15. Jphiego. Addressing long term demand in Kenya. PPIUCD at Nyeri PGH, KOGS; 2008.

16. Ndegwa S, Qureyshi Z, Lubano K. The impact of two levels of counseling on acceptance, uptake, early outcomes of post placental IUCD. University of Nairobi. Department of obstetrics and gynecology; 2010.

17. Lester F, Kakaire O, Byamugisha J, Averbach S, Fortin J, Maurer R. Intaceserean insertion of the copper T 380 a verses 6 week post cesarean insertion: a pilot study RCT. Contraception. 2015;91(3):198-203.

Cite this article as: Bedi PK, Guliani MS, Bala S.

A prospective study to assess the safety and expulsion rate of copper T380A in immediate postpartum period during caesarean section. Int $\mathbf{J}$ Reprod Contracept Obstet Gynecol 2016;5:3195-9. 